\title{
Temporal and spatial mapping of hand, foot and mouth disease in Sarawak, Malaysia
}

\author{
Noraishah M. Sham ${ }^{1}$, Isthrinayagy Krishnarajah ${ }^{1,2}$, Noor Akma Ibrahim ${ }^{1,2}$, Munn-Sann Lye ${ }^{3}$ \\ ${ }^{1}$ Laboratory of Computational and Operational Research, Institute for Mathematical Research, Universiti Putra \\ Malaysia, Serdang, Selangor, Malaysia; ${ }^{2}$ Department of Mathematics, Faculty of Science, Universiti Putra \\ Malaysia, Serdang, Selangor, Malaysia; ${ }^{3}$ Department of Community Health, Faculty of Medicine and Health \\ Sciences, Universiti Putra Malaysia, Serdang, Selangor, Malaysia
}

\begin{abstract}
Hand, foot and mouth disease (HFMD) is endemic in Sarawak, Malaysia. In this study, a geographical information system (GIS) was used to investigate the relationship between the reported HFMD cases and the spatial patterns in 11 districts of Sarawak from 2006 to 2012. Within this 7-years period, the highest number of reported HFMD cases occurred in 2006, followed by 2012, 2008, 2009, 2007, 2010 and 2011, in descending order. However, while there was no significant distribution pattern or clustering in the first part of the study period (2006 to 2011) based on Moran's I statistic, spatial autocorrelation $(\mathrm{P}=0.068)$ was observed in 2012 .
\end{abstract}

Keywords: hand, foot and mouth disease, geographical information systems, spatial autocorrelation, Malaysia.

\section{Introduction}

Hand, foot and mouth disease (HFMD) is endemic in the Malaysian state of Sarawak, which is located on the island of Borneo. The disease is commonly caused by a Coxsackie A virus and enterovirus 71 (EV71), but can also be caused by Coxsackie $\mathrm{B}$ and other enteroviruses. A sentinel surveillance conducted by Sarawak Health Department and the University of Malaysia in Sarawak (UNIMAS) has shown a 3-year epidemic cycle of EV71 (Sarawak Health Department, 2006). In Japan, the Infectious Disease Surveillance Center (IDSC) has reported a similar cyclical trend with predominantly EV71 isolation between 1983 and 2010 (IDSC, 2008).

The first outbreak of HFMD in Malaysia took place in 1997 in Sarawak. It affected mainly young children (aged $\leq 10$ years) and resulted in a number of deaths. Since then, the districts of Kuching, Sibu, Miri and Bintulu are noted hotspots for HFMD in Sarawak (Sarawak Health Department, 2006). Management protocols aimed at reducing transmission and the number of cases was initiated by the Health

\footnotetext{
Corresponding author:

Noraishah M. Sham

Laboratory of Computational and Operational Research

Institute for Mathematical Research

Universiti Putra Malaysia, 43400 Serdang, Selangor, Malaysia

Tel./Fax +6012 2870461

E-mail: aishahsham@yahoo.com
}

Department through various approaches, such as health education, specific legislation and interagency assistance (Sarawak Health Department, 2006).

Geographical information systems (GIS) have been widely used to visualise epidemiological data in terms of spread and clustering as well as ongoing trends. For an epidemiologist, this approach provides ample possibilities for mapping the spread of diseases and reveals the relationships with various parameters. Analytic methods based on GIS measure spatial patterns and evaluate potential spatial autocorrelations in order to see whether disease occurrences are clustered, dispersed or randomly spread. GIS have been used for various infectious diseases, e.g. Nakhapakorn and Jirakajohnkool (2006) who explored the potential of this technology for analysis of spatial factors with regard to dengue fever (DF) and dengue haemorrhagic fever (DHF), an Chaikaew et al. (2009) who revealed spatial patterns and hotspots of diarrhoea in the Chiang Mai province, Thailand between the years 2001 and 2006. The study by Dom et al. (2010) in Malaysia shows no significant distribution pattern for the dengue intensity index as the higher values tend to stay at the same locality throughout the year in Subang Jaya, Malaysia. However, to our knowledge, there have been no GIS studies on HFMD in Malaysia.

Here, we used GIS to investigate the spatial relationship between reported HFMD cases in Sarawak, Malaysia. Epidemiological data over a 7-year period provided by the Sarawak Health Department from 2006-2012 were used. This study was felt to be time- 
ly since HFMD is a serious infection, for which GIS had not been applied before, thus providing expectation of finding disease patterns that could indicate suitable control measures.

\section{Materials and methods}

\section{Study area}

Sarawak, located between latitude $0^{\circ} 50^{\prime}$ and $5^{\circ} \mathrm{N}$ and longitude $109^{\circ} 36^{\prime}$ and $115^{\circ} 40^{\prime} \mathrm{E}$ on the island of Borneo, is the largest state in Malaysia with a total area of $124,450 \mathrm{~km}^{2}$. The climate is warm throughout the year with heavy rainfall during the monsoon months from November to March. The study area covered 11 districts of Sarawak, as shown in Fig. 1.

\section{Data sources}

The HFMD data used in this study were compiled by the Crisis Preparedness and Response Centre, Communicable Disease Control Section, Sarawak Health Department on a weekly basis at the district level for seven years (2006-2012). This information is also available in Sarawak Weekly Epid News (Sarawak Health Department, 2012). The data were summarised by year and are presented in Table 1 .

\section{GIS procedure}

We used the Spatial Analyst tools available with ArcGIS software, version 10.1 (ESRI; Redlands, USA), which is in order to observe the spatial pattern of the disease. ArcMap was used to locate and present potential clustering of HFMD in the different districts in Sarawak. The HFMD dataset was classified into three classes depending on the number of cases reported per district and year, namely low $(<300)$, moderate (300$600)$ and high (>600).

\section{Statistical approach}

We aimed to find the risk measuring the frequency with which a disease occurs in a population over a period of time. This was done by building an equation (equation 1), where the incidence proportion was defined as the ratio of the number of reported HFMD cases $b$ to the population size $p$ as follows (CDC Atlanta, 2012):

$$
\text { Incidence proportion }=\left(h_{i} / p_{i}\right) \times 100,000
$$

(equation 1)

where $i$ represent the years $\mathrm{i}=1,2, \ldots, 7$.

The spatial autocorrelation tool in ArcGIS calculates the value of Moran's I giving both a $Z$ score and Pvalue that gives the significance of the index. Generally, this index varies between 1.0 and -1.0, where values near 1.0 indicate clustering and the other extreme random dispersion. The Moran's I statistic for spatial autocorrelation (Mitchell, 2005) is given as

$$
I=\frac{n}{S_{0}} \frac{\sum_{i=1}^{n} \sum_{i=1}^{n} w_{i j} z_{i} z_{j}}{\sum_{i=1}^{n} z_{i}^{2}}
$$

(equation 2)

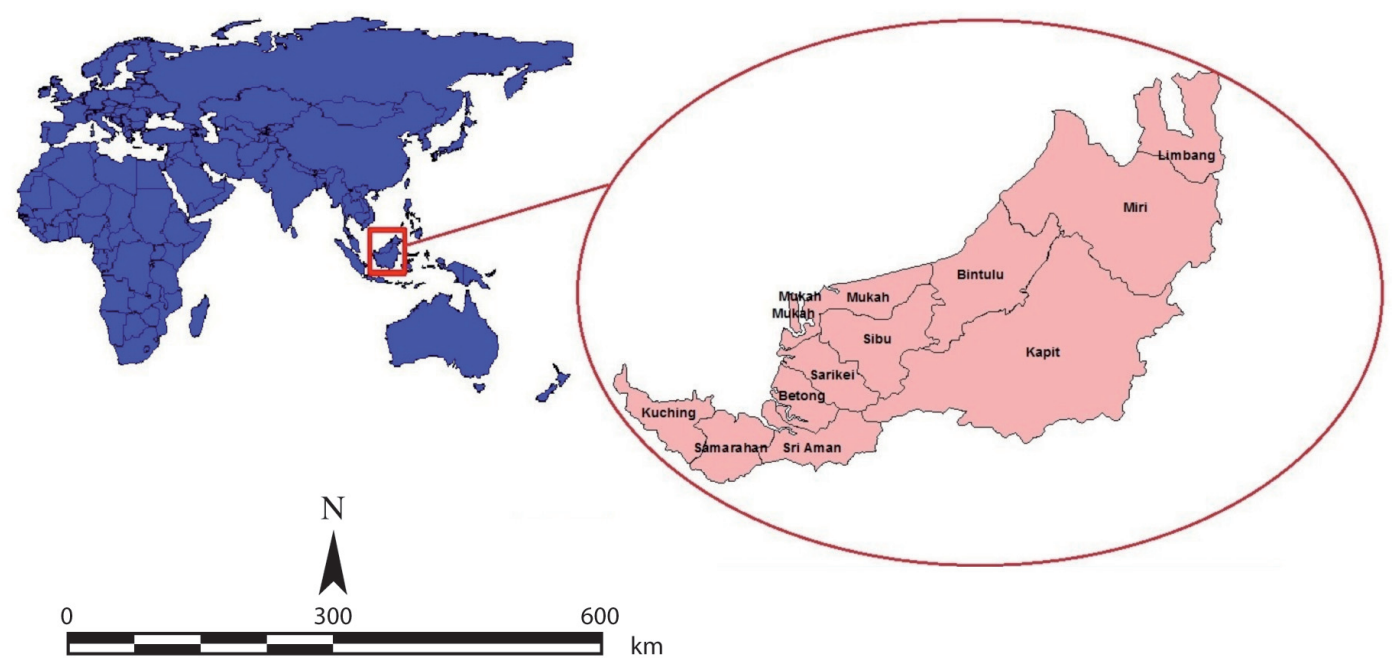

Fig. 1. Study area, Sarawak, Malaysia. 
Table 1. Reported cases of HFMD in Sarawak 2006-2012.

\begin{tabular}{lrrrrrrr}
\hline District & 2006 & 2007 & 2008 & 2009 & 2010 & 2011 & 2012 \\
\hline Betong & 269 & 118 & 292 & 200 & 141 & 77 & 663 \\
Bintulu & 1,887 & 883 & 1,427 & 944 & 657 & 760 & 938 \\
Kapit & 542 & 240 & 308 & 762 & 155 & 106 & 596 \\
Kuching & 2,350 & 2,015 & 3,298 & 1,536 & 1,256 & 769 & 4,571 \\
Limbang & 487 & 55 & 283 & 69 & 110 & 21 & 557 \\
Miri & 3,107 & 877 & 1,628 & 1,826 & 1,016 & 323 & 1,354 \\
Mukah & 858 & 207 & 242 & 177 & 411 & 126 & 501 \\
Samarahan & 736 & 379 & 601 & 325 & 196 & 198 & 1,755 \\
Sarikei & 961 & 348 & 325 & 373 & 322 & 234 & 969 \\
Sibu & 3,361 & 977 & 1,600 & 3,103 & 578 & 171 & 1,024 \\
Sri Aman & 317 & 472 & 431 & 340 & 168 & 115 & 567 \\
Total & 14,875 & 6,571 & 10,435 & 9,655 & 5,010 & 2,900 & 13,495 \\
\hline
\end{tabular}

where $z_{i}$ is the deviation of an attribute for feature $i$ from its mean $\left(x_{i}-\bar{X}\right), z_{j}$ the deviation of the variable of interest with respect to the mean $\left(x_{j}-\bar{X}\right), w_{i, j}$ the spatial weight between feature $i$ and $j, n$ the total number of features, and $S_{0}$ the aggregate of all the spatial weights:

$$
S_{0}=\sum_{i=1}^{n} \sum_{j=1}^{n} w_{i j}
$$

(equation 3)

The $Z_{I}$-score for the statistic is computed as given as

$$
Z_{I}=\frac{I-E[I]}{\sqrt{V[I]}}
$$

(equation 4)

where $E[I]=-1 /(n-1)$ and $V[I]=\mathrm{E}\left[I^{2}\right]-\mathrm{E}[I]^{2}$

\section{Results}

As seen in Table 1 and Fig. 2, the HFMD outbreaks occurred in a cyclical pattern approximately every two to three years in the 7-year study period. In retrospect, 2006 held the highest total number of reported cases, followed by 2012, 2008, 2009, 2007, 2010 and 2011.

The number of reported cases showed fluctuations over the study period with a first increase between weeks 9 to 13 of the year, i.e. about March (Fig. 2) followed by a levelling off without any obvious further peaks in most cases, except in the years 2006, 2008 and 2012, when second peaks were produced around weeks 30, 33 and 25, respectively. In 2008, however, there was a final steep increase from week 40 producing a third peak reaching 607 cases by the end of the year, i.e. between weeks 41 and 50. Overall, there were comparatively fewer reported cases showing severe seasonal outbreaks between March and May over the whole study period.

The incidence rate for 2006 was the highest compared to the rest as shown in Table 2. The rate shows that there were about 631 cases for every 100,000 people in Sarawak in that year. Although the absolute number of reported cases was comparatively high for the years 2006 and 2012 (Table 1) and the incidence rates comparatively high for 2006, 2008, 2009 and 2012 (Table 2), Moran's I was only positive for 2011 and 2012. Looking at the Z-score, there were only small variations indicating random distribution, except for the year 2012, which was the only year that showed statistical significance $(\mathrm{P}=0.068)$.

The spatial distribution of the reported cases by district can be visualised using the ArcMap tool in ArcGIS that is capable of showing the different val-

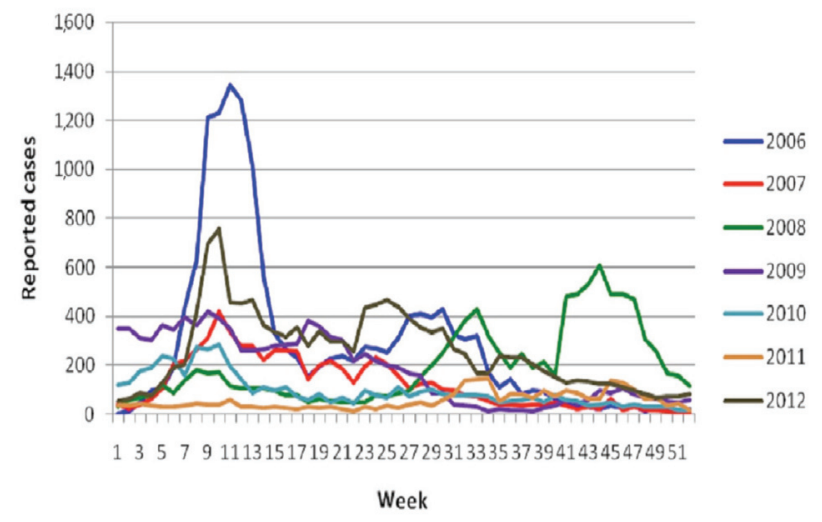

Fig. 2. Weekly reported cases of HFMD between 2006 and 2012 in Sarawak, Malaysia. 
Table 2. Spatial autocorrelation of HFMD cases in Sarawak, 2006-2012.

\begin{tabular}{|c|c|c|c|c|c|c|c|}
\hline & 2006 & 2007 & 2008 & 2009 & 2010 & 2011 & 2012 \\
\hline Incidence proportion* & 630.97 & 273.34 & 425.43 & 385.64 & 199.88 & 115.25 & 530.09 \\
\hline Moran's $I$ & -0.1061 & 0.1086 & 0.0424 & -0.3130 & -0.0257 & 0.0184 & 0.2175 \\
\hline Z (Moran) & -0.0245 & 0.0395 & 0.2582 & -0.9361 & 0.3075 & 0.5008 & 1.8260 \\
\hline P-value & 0.981 & 0.969 & 0.796 & 0.349 & 0.759 & 0.617 & 0.068 \\
\hline
\end{tabular}

*Per 100,000 population.

ues' association with the geographical areas. As seen in Fig. 3, no specific HFMD patterns were discerned among the 11 districts for the years 2006, 2007, 2008, 2010 and 2011 except in 2009 and 2012, where the HFMD cases seem to be clustered covering neighbouring districts in the centre of the state. In 2012, the disease was not only clustered in the centre, but this pattern continued along the districts in the south-west, including Kuching, the most western district.

\section{Discussion}

The data presented here show that HFMD follows a seasonal pattern with most outbreaks between March and May based on the number of weekly reported cases. This pattern follows the situation in Singapore, where surveillance data from 2002-2009 showed similar annual peaks between March to May (Ministry of Health Singapore, 2010).

In this study, although visually cases seem to be clustered covering neighbouring districts in the centre of the state in the year 2009 and 2012, based on the $\mathrm{P}$-value we must conclude that spatial autocorrelation existed only in 2012. Although the P-value was not strong, it stood out in comparison with the other years covered by the study, and it clearly indicted statistical significance of the results. This study covered only the districts in Sarawak due to the limited availability of surveillance data and also the period of study covered is only 7 years. In future, it is hoped that a study for the whole country could be carried out.
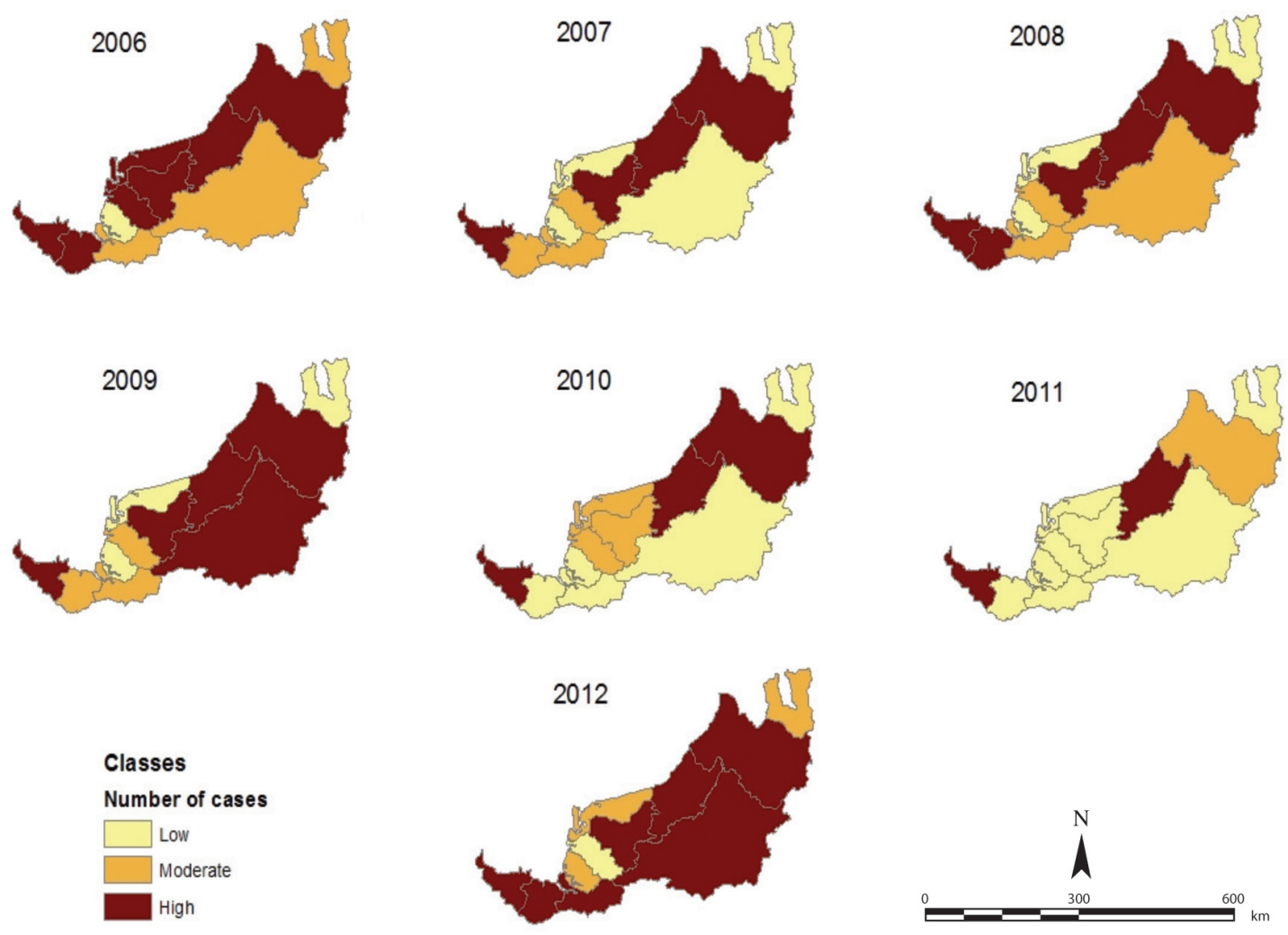

Fig. 3. Mapping of spatial autocorrelation based on number of cases by districts in Sarawak. 


\section{Conclusion}

An approach based on Moran's I statistic complemented with ArcMap for visualisation facilitates decision-making regarding various options such as isolation according to districts and implement general control measures in more disease prone districts to control the spread of HFMD during outbreaks. It also helps to choose the right timing and strategy for the implementation of control activities.

\section{Acknowledgements}

Special thanks to Crisis Preparedness and Response Centre, Communicable Disease Control Section, Sarawak Health Department for the invaluable assistance in compiling the seven years dataset for us in weekly routine; many thanks to the Institute for Medical Research and Ministry of Science, Technology and Innovation for the financial support. This project was funded by the Ministry of Higher Education under the Fundamental Research Grant Scheme.

\section{References}

CDC Atlanta, 2012. Principles of epidemiology in public health practice (third edition). Available at http://www.cdc.gov/ osels/scientific_edu/ss1978/lesson3/section2.html (accessed on March 2014).
Chaikaew N, Tripathi NK, Souris M, 2009. Exploring spatial patterns and hotspots of diarrhea in Chiang Mai, Thailand. Int J Health Geogr 8, 36-36.

Dom N, Ahmad A, Nasir R, Ismail R, 2010. Spatial mapping of temporal risk characteristic of dengue cases in Subang Jaya. Proceedings of the International Conference Of Science And Social Research. Research Management Institute, University technology MARA, 361-366 pp.

IDSC, 2008. Isolation and detection report of viruses by year, 1982-2008: enterovirus 1 and enterovirus 2. Available at Infectious Disease Surveillance Center. Available at: http://idsc.nih.go.jp/iasr/index.html (accessed on July 2013).

Ministry of Health Singapore, 2010. Air/droplets-borne diseases

- Hand, foot and mouth disease. Weekly Infectious Disease Bulletin Singapore 2002-2009. Available at http://www. moh.gov.sg/content/moh_web/home/statistics/infectiousDiseas esStatistics/weekly_infectiousdiseasesbulletin.html (accessed on July 2013).

Mitchell A, 2005. The ESRI Guide to GIS Analysis. ESRI Press.

Nakhapakorn K, Jirakajohnkool S, 2006. Temporal and spatial autocorrelation statistics of dengue fever. Dengue Bull 30, 177-183.

Sarawak Health Department, 2006. HFMD in Sarawak. Available at http://jknsarawak.moh.gov.my/en/modules/ mastop_publish/?tac=122 (accessed on June 2013).

Sarawak Health Department, 2012. Sarawak Weekly Epid News. Available at http://jknsarawak.moh.gov.my (accessed on July 2013). 